\title{
THE INFRARED ARRAY CAMERA DARK FIELD: FAR-INFRARED TO X-RAY DATA
}

\author{
J. E. Krick ${ }^{1}$, J. A. Surace ${ }^{1}$, D. Thompson ${ }^{2}$, M. L. N. Ashby ${ }^{3}$, J. Hora ${ }^{3}$, V. Gorjian ${ }^{4}$, L. Yan ${ }^{1}$, D. T. Frayer ${ }^{5}$, E. Egami ${ }^{6}$, \\ AND M. LACY ${ }^{1}$ \\ ${ }^{1}$ Spitzer Science Center, MS 220-6, California Institute of Technology, Jet Propulsion Laboratory, Pasadena, CA 91125, USA; jkrick@caltech.edu \\ ${ }^{2}$ Large Binocular Telescope Observatory, University of Arizona, Tucson, AZ 85721, USA \\ ${ }^{3}$ Harvard-Smithsonian Center for Astrophysics, 60 Garden Street, Cambridge, MA 02138, USA \\ ${ }^{4}$ Jet Propulsion Laboratory, California Institute of Technology, Pasadena, CA, 91109, USA \\ ${ }^{5}$ NASA Herschel Science Center, California Institute of Technology, Pasadena, CA 91109, USA \\ ${ }^{6}$ Department of Astronomy, University of Arizona, 933 N. Cherry Avenue, Tucson, AZ 85721, USA \\ Received 2009 April 16; accepted 2009 September 11; published 2009 October 14
}

\begin{abstract}
We present 20 band photometry from the far-IR to X-ray in the Spitzer Infrared Array Camera (IRAC) dark field. The bias for the near-IR camera on Spitzer is calibrated by observing a $\sim 20^{\prime}$ diameter "dark" field near the north ecliptic pole roughly every two-to-three weeks throughout the mission duration of Spitzer. The field is unique for its extreme depth, low background, high quality imaging, time-series information, and accompanying photometry including data taken with Akari, Palomar, MMT, KPNO, Hubble, and Chandra. This serendipitous survey contains the deepest mid-IR data taken to date. This data set is well suited for studies of intermediateredshift galaxy clusters, high-redshift galaxies, the first generation of stars, and the lowest mass brown dwarfs, among others. This paper provides a summary of the data characteristics and catalog generation from all bands collected to date as well as a discussion of photometric redshifts and initial and expected science results and goals. To illustrate the scientific potential of this unique data set, we also present here IRAC color-color diagrams.
\end{abstract}

Key words: cosmology: observations - galaxies: photometry

\section{INTRODUCTION}

Deep, multi-wavelength surveys are an important tool in studying the formation and evolution of galaxies. Infrared data are particularly useful in these surveys. Near-IR wavelengths provide a direct measurement of the stellar content of galaxies in the absence of a dominant active galactic nucleus (AGN) contribution at redshifts as high as 3 . The longer wavelengths sample emission primarily from polycyclic aromatic hydrocarbons (PAHs), as well as direct thermal emission from hot dust. These are markers of star-forming galaxies and AGNs.

The dark field is a multi-wavelength survey based on observations carried out as part of the calibration for the Infrared Array Camera (IRAC; Fazio et al. 2004) on the Spitzer Space Telescope (Werner et al. 2004). Rather than being designed around specific science objectives, like many other Great Observatory surveys (HDF, CDF, SWIRE, GOODS, GEMS, COSMOS, etc.; williams et al. 1996; Giacconi et al. 2001; Lonsdale et al. 2003; Giavalisco et al. 2004; Rix et al. 2004; Scoville et al. 2007), the survey area and observation strategy were selected based on the calibration requirements for IRAC. Observations were taken to measure the dark current and biases of the four detector arrays (see Section 2.1.1) near the beginning and end of each IRAC observing period (or "campaign"), resulting in 2-5 dark field measurements every two-to-three weeks throughout the entire duration of the mission. Each of these observations in the dark field collects roughly $2 \mathrm{hr}$ of integration time total in each of the four bands in a single $5^{\prime} \times 5^{\prime}$ field of view. Each new visit is observed in a slightly different position due to spacecraft orientation and rotation. The center of the field is located at $17^{\mathrm{h}} 40^{\mathrm{m}}$, $+69^{\mathrm{d}}$ (J2000), very near the north ecliptic pole (NEP). Over the course of the mission, the observations have filled in a region roughly $20^{\prime}$ in diameter. This has created the deepest ever midIR survey, exceeding the depth of the deepest planned regular Spitzer surveys over several times their area. Furthermore, this is the only field for which a 5+year baseline of mid-IR periodic observations exists (see Frost et al. 2009, for more information on the time-series data).

The resulting observations are unique in several ways. The dark field lies in the lowest possible region of zodiacal background, the primary contributor to the infrared background at these wavelengths, and as such is in the region where the greatest sensitivity can be achieved in the least amount of time. The area was also chosen specifically to be free of bright stars and very extended galaxies, which allows clean imaging to very great depth. The field lies in the northern continuous viewing zone, a $10^{\circ}$ radius region centered on the NEP that is always visible to Spitzer, which means many opportunities exist to re-observe the field essentially continuously. The observations are done at many position angles (which are a function of time of observation) leading to a more uniform, circularized, final point-spread function (PSF). Finally, because the calibration data are taken directly after anneals, they are more free of electronic artifacts such as hot pixels and latent images than ordinary guest observer (GO) observations.

To enhance the IRAC dark field data set we have obtained imaging data in 16 other bands including Palomar/MMT/ KPNO $u^{\prime}, g^{\prime}, r^{\prime}, i^{\prime}, z^{\prime}, J, H, K$, Hubble Space Telescope (HST)/Advanced Camera for Surveys (ACS) F814W, Akari $4,11,15$, and $18 \mu \mathrm{m}$, Spitzer MIPS 24 and $70 \mu \mathrm{m}$, and Chan$d r a$ ACIS-I. Although the entire dark field is $>20^{\prime}$ in diameter, because of spacecraft dynamics the central $\sim 15^{\prime}$ is significantly deeper and freer of artifacts. Therefore, it is this area which we have matched with the additional observations. All spacebased data sets are publicly available through their respective archives.

This paper is structured in the following manner. In Section 2, we present the data for all 20 bands, its reduction as well as source extraction and photometry. Details of the composite catalog are presented in Section 3. Photometric redshifts are 
Table 1

Observational Data

\begin{tabular}{|c|c|c|c|c|c|c|c|}
\hline $\begin{array}{c}\text { Telescope } \\
\text { Instrument }\end{array}$ & Waveband & $\begin{array}{l}\text { Central } \lambda \\
\qquad(\mu \mathrm{m})\end{array}$ & $\begin{array}{l}\text { Avg. Exptime } \\
\text { (hours/pixel) }\end{array}$ & $\begin{array}{c}\text { Area } \\
\left(\square^{\prime}\right)\end{array}$ & $\begin{array}{l}3 \sigma \text { Depth } \\
\text { (AB magnitude) }\end{array}$ & $(\mu \mathrm{Jy})$ & $\begin{array}{l}\text { Avg. FWHM } \\
\text { (arcsec) }\end{array}$ \\
\hline Chandra ACIS-I & & $2-8 \mathrm{KeV}$ & 28.0 & 290 & $3.6 \times 10^{-16 c}$ & & 5.0 \\
\hline Palomar Hale LFC & $u^{\prime}$ & 0.35 & 6.0 & 450 & 27.2 & 0.05 & 1.0 \\
\hline Palomar Hale LFC & $g^{\prime}$ & 0.47 & 6.2 & 450 & 27.1 & 0.05 & 1.3 \\
\hline Palomar Hale LFC & $r^{\prime}$ & 0.63 & 4.1 & 450 & 26.6 & 0.09 & 0.9 \\
\hline Palomar Hale LFC & $i^{\prime}$ & 0.77 & 4.7 & 450 & 26.0 & 0.15 & 1.2 \\
\hline HST ACS & F814W & 0.81 & 1.4 & 260 & 28.6 & 0.01 & 0.1 \\
\hline MMT Megacam & $z^{\prime}$ & 0.91 & 3.6 & 580 & 25.9 & 0.16 & 1.0 \\
\hline NOAO Mayall Flamingos & $J$ & 1.20 & 0.5 & 220 & 21.4 & 10.2 & 1.2 \\
\hline MMT SWIRC & $J$ & 1.2 & 5.2 & 25 & 24.4 & 0.65 & 0.8 \\
\hline Palomar Hale WIRC & $J$ & 1.25 & 0.8 & 120 & 21.8 & 7.1 & 1.1 \\
\hline MMT SWIRC & $H$ & 1.6 & 0.2 & 225 & 23.0 & 2.4 & 0.8 \\
\hline Palomar Hale WIRC & $H$ & 1.63 & 0.7 & 120 & 22.1 & 5.4 & 1.1 \\
\hline Palomar Hale WIRC & $K$ & 2.15 & 1.6 & 210 & 21.2 & 12.3 & 1.1 \\
\hline Spitzer IRAC & & 3.6 & 8.0 & 380 & 25.7 & $0.2^{\mathrm{d}}$ & 1.9 \\
\hline Spitzer IRAC & & 4.5 & 10.0 & 380 & 25.9 & $0.17^{\mathrm{d}}$ & 1.9 \\
\hline Akari IRC & & 4.5 & 1.8 & 100 & 23.5 & 1.5 & 4.4 \\
\hline Spitzer IRAC & & 5.8 & 8.0 & 380 & 26.3 & $0.11^{\mathrm{d}}$ & 1.9 \\
\hline Spitzer IRAC & & 8.0 & 10.0 & 380 & 26.3 & $0.11^{\mathrm{d}}$ & 2.1 \\
\hline Akari IRC & & 11 & 2.4 & 100 & 21.0 & 15 & 6.2 \\
\hline Akari IRC & & 15 & 1.0 & 100 & 20.1 & 33 & 6.5 \\
\hline Akari IRC & & 18 & 0.8 & 100 & 19.8 & 45 & 6.7 \\
\hline Spitzer MIPS & & 24 & 1.0 & 225 & 20.8 & 17.3 & 5.8 \\
\hline Spitzer MIPS & & 70 & 1.6 & 160 & 14.8 & 3200 & 18.6 \\
\hline
\end{tabular}

Notes.

a Total and maximum exposure times are presented in Section 2.

${ }^{b}$ Average FWHM as measured from a number of point sources in the final image.

c $\operatorname{erg~s}^{-1} \mathrm{~cm}^{-2}$.

d IRAC depths are given as measured 95\% completeness limits, see Section 2.1.1.

derived and errors are calculated in Section 4. Lastly, a small discussion of the science goals are presented in Section 5. Throughout this paper we use $H_{0}=70 \mathrm{~km} \mathrm{~s}^{-1} \mathrm{Mpc}^{-1}, \Omega_{M}=$ $0.3, \Omega_{\Lambda}=0.7$. All photometry is quoted in the $\mathrm{AB}$ magnitude system.

\section{DATA}

The details of our data set are summarized in Table 1 and in the sections below. Data are presented roughly in order of decreasing wavelength with the exception that the IRAC data are discussed first. Figure 1 shows the filter curves of the optical through infrared bands discussed below. Figure 2 shows a representative sample of data thumbnails.

\subsection{Spitzer Space Telescope \\ 2.1.1. IRAC}

IRAC simultaneously observes with four detectors at 3.6, $4.5,5.8$, and $8.0 \mu \mathrm{m}$. Each detector has a 5.2 $\times 5.2$ field of view with 1."2 pixels. Images are acquired in pairs (3.6 and $5.8 \mu \mathrm{m}$, 4.5 and $8.0 \mu \mathrm{m}$ ) of two adjacent fields, separated by 6.5 , using beam splitters.

This work is based on a preliminary combination of calibration data taken from 2003 December to 2005 January; $\approx 30 \%$ of the expected dark field depth, not including the warm mission (see Figure 3). The 3.6, 4.5, 5.8, and $8.0 \mu \mathrm{m}$ data have a total exposure time of $40 \mathrm{hr}$ per filter covering $380 \mathrm{arcmin}^{2}$ to limiting depths of $0.2,0.17,0.11,0.11 \mu \mathrm{Jy}$, respectively. The maximum per pixel exposure time is $28 \mathrm{hr}$ at 3.6 and $5.8 \mu \mathrm{m}$ and $21 \mathrm{hr}$ at 4.5 and $8.0 \mu \mathrm{m}$. The median exposure time per pixel is $8-10 \mathrm{hr}$, see below. Raw astrometry from the Spitzer
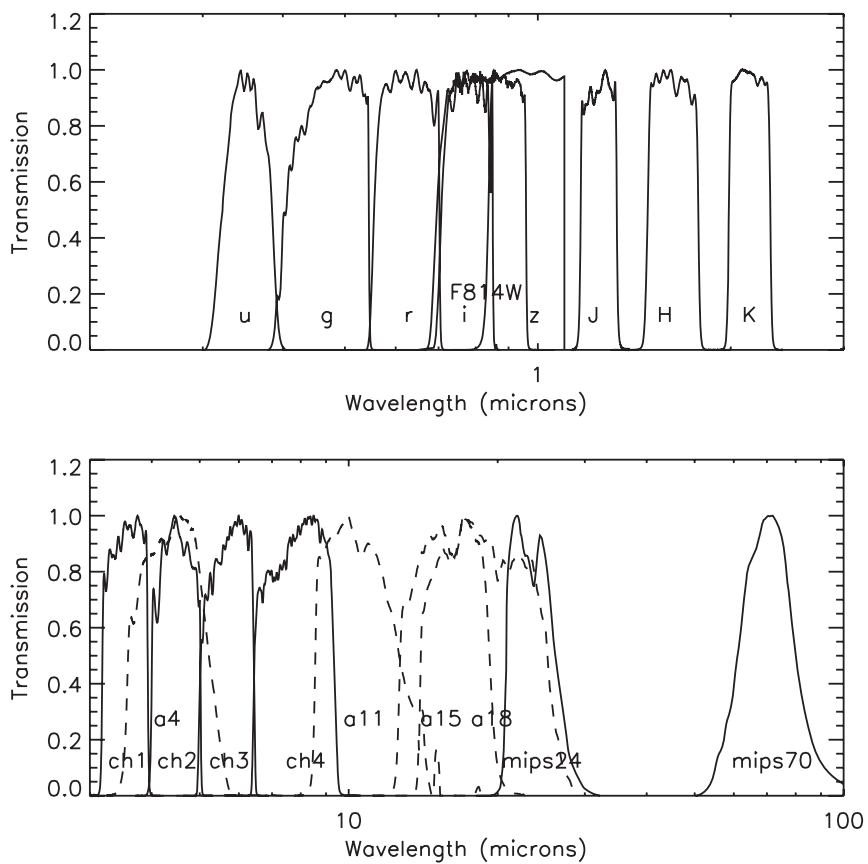

Figure 1. Filter transmission curves for the optical to far-IR bands. All curves are normalized for comparison. The upper plot shows optical through near-IR. Of the near-IR bands, only the Palomar WIRC $J, H$, and $K$ curves are shown for clarity. The lower plot shows the Spitzer and Akari (dashed lines) mid-IR to far-IR bands.

pointing control system, based on inputs from the star tracker and gyroscopes, is used as the input to a pointing refinement program. The absolute positions of stars from the Two Micron 

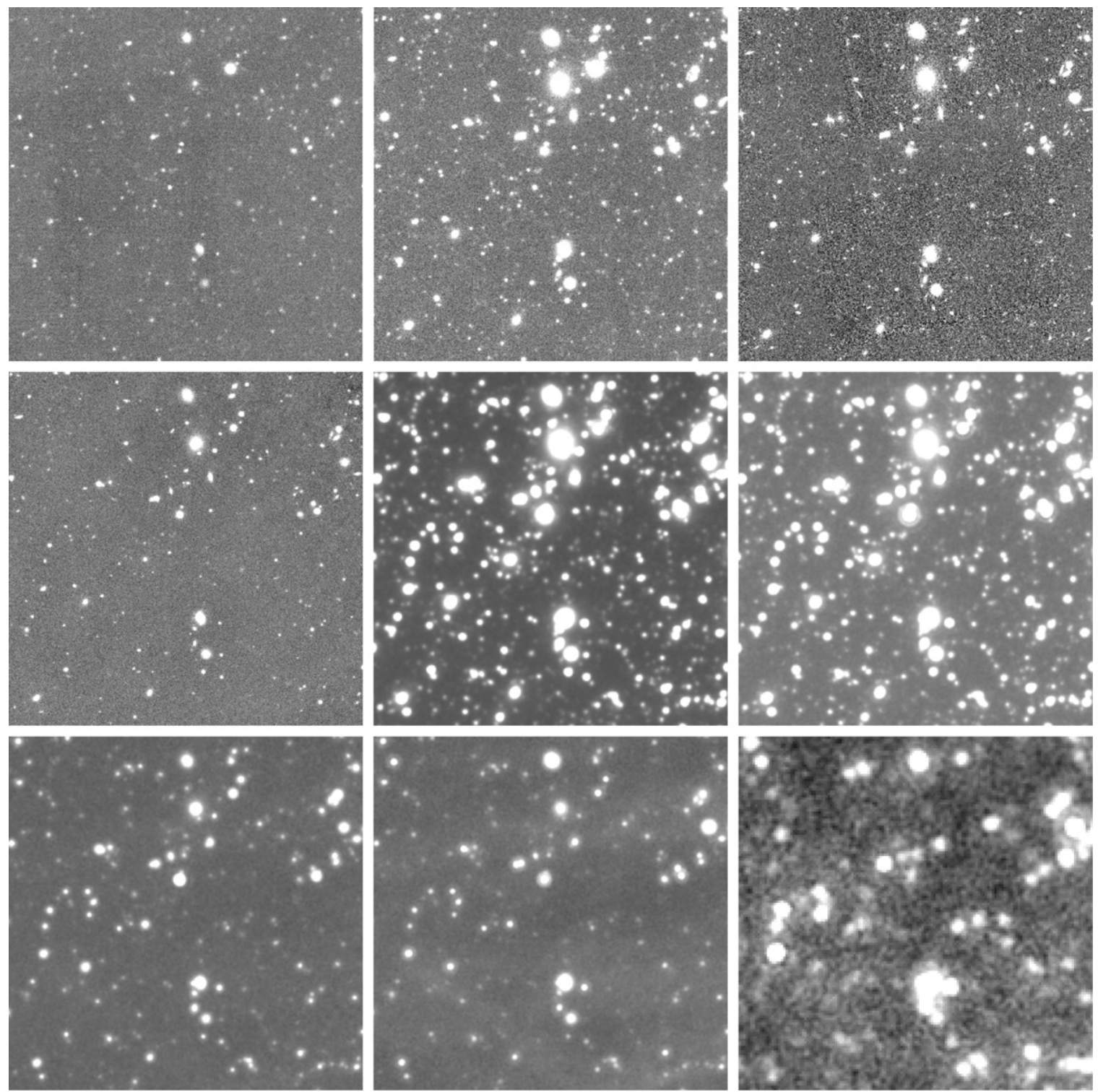

Figure 2. Representative images of the field, $3 \mathrm{arcmin}$ on a side. The order of the images is $u^{\prime}, r^{\prime}, H S T / \mathrm{ACS}$ F814W, J, 3.6, 4.5, 5.8, 8.0, $24 \mu \mathrm{m}$.

All Sky Survey (2MASS) along with the relative positions of stars common to different individual IRAC frames are then used to improve the astrometry from the raw spacecraft astrometry. Final astrometric accuracy for an individual frame is $\sim 0 ! 3 \mathrm{rms}$. Because field rotation during the mission should average out many systematic effects, the final astrometric accuracy of the IRAC dark field mosaic is expected to be better than this. The Basic Calibrated Data (BCD) products produced by the Spitzer Science Center (pipeline versions S11, S12, S13) were further reduced using a modified version of the pipeline developed for the Spitzer Wide area Infrared Extragalactic survey (SWIRE) (Surace et al. 2005). This pipeline primarily corrects image artifacts and forces the images onto a constant background (necessitated by the continuously changing zodiacal background as seen from Spitzer). The data were co-added onto a regularized 0' 6 grid using the MOPEX software developed by the Spitzer Science Center (SSC) as described in Makovoz \& Marleau (2005). Experiments we performed with DAOPHOT demonstrate that nearly all extragalactic sources are at least marginally resolved by IRAC, particularly at shorter wavelengths, and hence point source fitting is inappropriate for photometry.
Photometry is done using the high spatial resolution ACS data as priors for determining an individual, object-specific aperture shape for extracting the IRAC fluxes. The advantages of this are deeper photometry and more accurate color measurements between low- and high-resolution observations. Because the IRAC beam is so large (compared to ACS), using the higher resolution data for shape information will allow us to deblend otherwise overlapping sources and therefore do photometry to fainter limits (below the confusion limit) than with IRAC alone. The disadvantage of using this technique is that we must assume that the galaxy morphology is the same across the wavelength range of the high- to low-resolution data. This assumes that ACS F814 light is co-spatial with IR light. At high redshifts, this assumption will be reasonable because in those galaxies we cannot resolve any age or star formation (SF) gradients across the galaxy. For low-redshift galaxies we rely on the RayleighJeans side of the spectral energy distribution (SED) being less sensitive to age so that F814 and IRAC are measuring the same population of stars. This assumption is not valid for all galaxies and must be considered when using the catalog for science goals. The use of high-resolution data as priors for low-resolution 

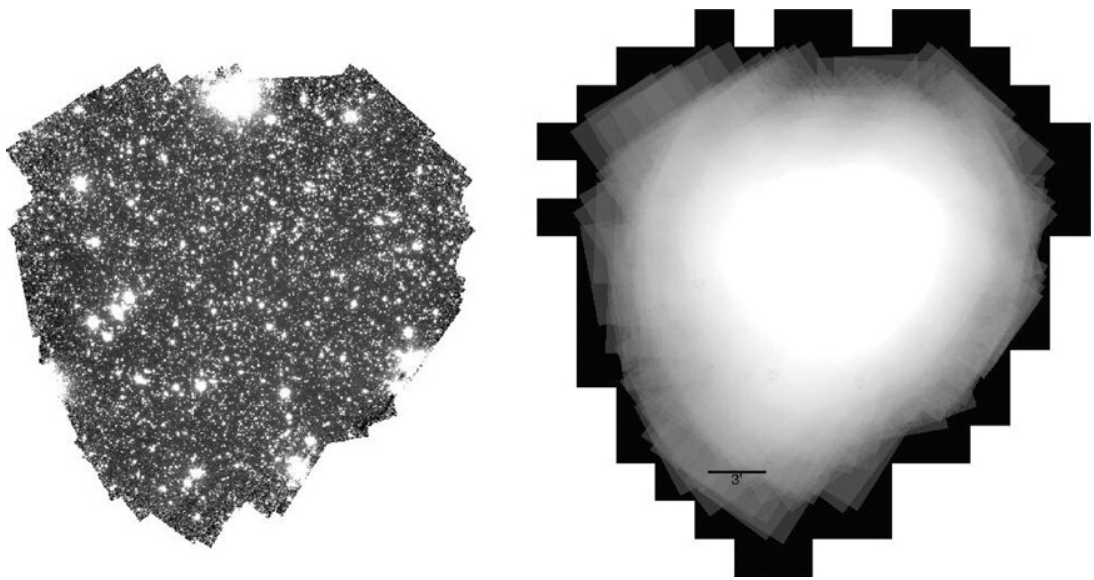

Figure 3. Left: the full $3.6 \mu \mathrm{m}$ data image. Right: gray-scale coverage map of the $3.6 \mu \mathrm{m}$ data with gray scale ranging from 1 to 1000 images, each of $100 \mathrm{~s}$ exposure time. north is up, east is to the right. The actual NEP is 3.5 away.

photometry has been used on similar data sets in the literature (Fernández-Soto et al. 1999; Papovich et al. 2001; Shapley et al. 2005; Grazian et al. 2006; Laidler et al. 2007).

This method begins by first running source detection and photometric extraction on the co-added IRAC images using a matched filter algorithm with image backgrounds determined using the mesh background estimator in SExtractor (Bertin \& Arnouts 1996). This catalog is merged with the HST/ACS catalog. For every object in that catalog, if the object is detected in ACS then we use the ACS shape parameters to determine the elliptical aperture size for the IRAC images. ACS shape parameters are determined by SExtractor on isophotal object profiles after deblending, such that each ACS pixel can only be assigned to one object (or the background). For objects which are not detected in ACS, but which are detected in IRAC, we simply use the original IRAC SExtractor photometry. Because of the larger IRAC beam, for all objects in the catalog we impose a minimum semi-major axis of $2^{\prime \prime}$. In all cases aperture corrections are computed individually from PSFs provided by the SSC based on the aperture sizes and shapes used for photometry. ${ }^{7}$

Final aperture photometry was performed using custom extraction software written in IDL and based on the APER and MASK_ELLIPSE routines with the shape information from SExtractor, from either ACS or IRAC as described above, using local backgrounds. Fluxes are measured in all IRAC bands at the location of all ACS detections without applying a flux limit (IRAC detection limits are discussed below). Because we use local backgrounds, the measured fluxes of objects near the confusion limit should have a larger scatter than those nonconfused objects, but will on average be the correct flux. This will not effect the photometric redshifts, as it will likely shift all IRAC points up or down, but not relative to each other. Figure 4 shows the difference between using the IRAC photometry alone and using the ACS-based photometry as described here. This plot is a direct analog of Figure 2 in Laidler et al. (2007) which shows the comparison of fluxes for the software package TFIT. We do not see the systematic offset in fluxes that they see at low flux levels.

Determining the detection limits of the IRAC data is complicated by varying exposure times across the field, source confusion, and our use of ACS locations as priors for photometry. Field coverage is a complex function of spacecraft dynamics (see Figures 3 and 5). Within the central $180 \operatorname{arcmin}^{2}$ of the

\footnotetext{
7 http://ssc.spitzer.caltech.edu/irac/psf.html
}

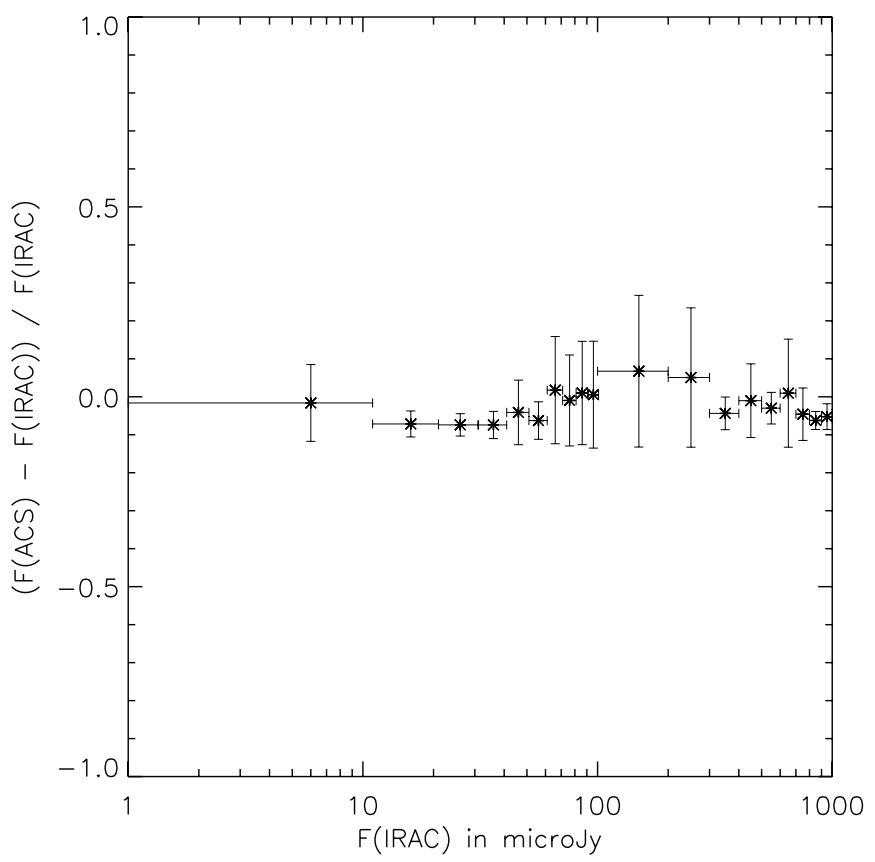

Figure 4. Difference between ACS based (F(ACS)) and IRAC-alone based (F(IRAC)) photometries for the 18,300 sources in common in the catalog with flux measurements in greater than five wavebands. Points and error bars show the biweight center and dispersion of the distribution within each bin. This allows for robust outlier rejection. Note that the bin size changes with the flux.

field, the median exposure time per pixel is $8 \mathrm{hr}$ at 3.6 and $5.8 \mu \mathrm{m}$ and $10 \mathrm{hr}$ at 4.5 and $8.0 \mu \mathrm{m}$. The maximum exposure time in the center is $28 \mathrm{hr}$ and $21 \mathrm{hr}$ at 3.6 and 5.8 and 4.5 and $8.0 \mu \mathrm{m}$, respectively. A total of $40 \mathrm{hr}$ of observations went into covering the entire field. The confusion limit in the IRAC data arises because both the PSF ( 2 "FWHM) and the number of sources are large. We have used ACS priors to be able to deblend confused sources, and therefore our detection limit is below the nominal confusion limit. One effect of using ACS for deblending is that the depth of our photometry will be color dependent. We have IRAC photometry below the confusion limit for all objects which are detected in the F814W band, but cannot deblend objects for which there is no F814W counterpart. Therefore, our photometry is deeper for sources which have bluer spectral shapes, whereas it is confusion limited for the redder, IRAC only detections. Because of these three complexities, there is no one single value for the detection limit of the survey. 


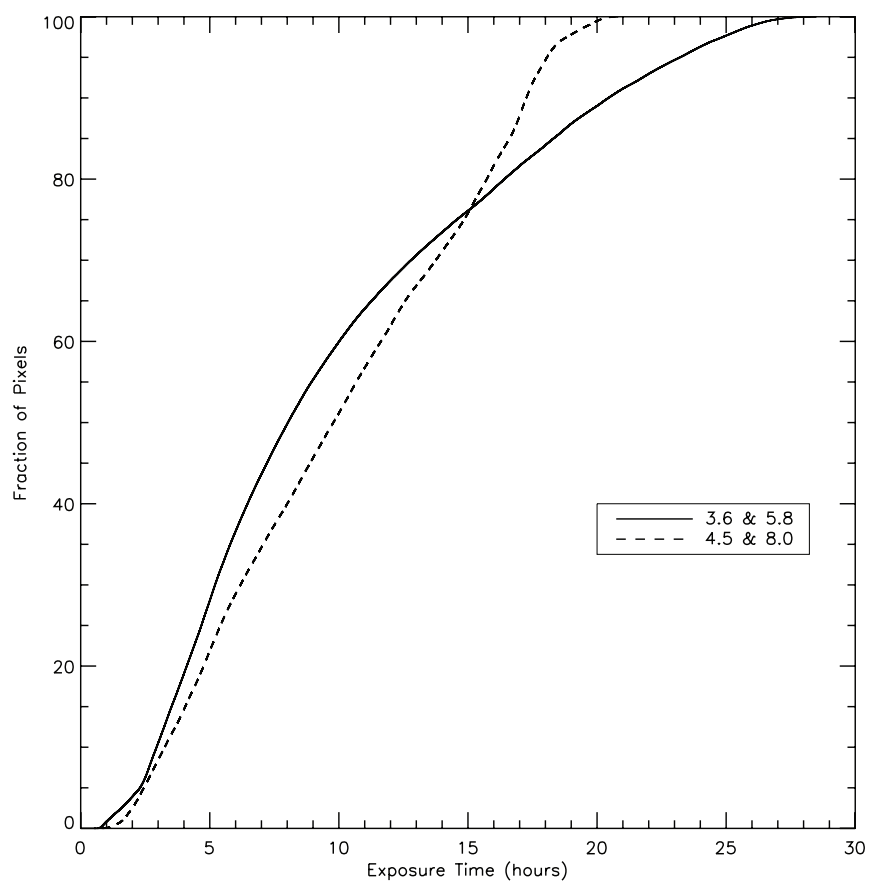

Figure 5. Cumulative distribution of the number of pixels vs. exposure time in hours for the central $15^{\prime}$ diameter region. The solid line shows the coverage for the 3.6 and $5.8 \mu \mathrm{m}$ images, the dashed line is for 4.5 and $8.0 \mu \mathrm{m}$ images.

We are able to measure nominal $95 \%$ completeness limits in the IRAC passbands from a number count diagram by measuring where the number of observed objects drops below $95 \%$ of the expected number of objects. The expected numbers are calculated by fitting a straight line to the brighter flux number counts and assuming that the faint number counts would also follow that line in the absence of confusion. Figure 6 shows the number counts in the four IRAC bands with a best-fit line to the detections to show where the number of measured fluxes drops below $95 \%$ of the expected numbers. The figure also shows the larger confusion in the shorter wavelength bands. The measured and expected number counts are lower in the longer wavelength bands. Final values at 3.6, 4.5, 5.8, and $8.0 \mu \mathrm{m}$ are 0.2, 0.17, 0.11 , and $0.11 \mu \mathrm{Jy}$, respectively (see Table 1 ). A more complete analysis of the number counts will be presented in a future paper.

\subsubsection{MIPS $70 \mu \mathrm{m}$}

The $70 \mu \mathrm{m}$ observations were taken with the Multiband Imaging Photometer for Spitzer (MIPS; Rieke et al. 2004) with a total exposure time per pixel of $1.6 \mathrm{hr}$ covering $160 \mathrm{arcmin}^{2}$ to a limiting $4 \sigma$ depth of $4.3 \mathrm{mJy}$ (Program ID 30222). The data were taken as a $5 \times 2$ grid of pointings $(5.3 \times 2$ ' 6 field of view) in small-scale photometry mode with $10 \mathrm{~s}$ exposures. The raw $70 \mu \mathrm{m}$ data were downloaded from the SSC archive and were reduced using the Germanium Reprocessing Tools (GeRT, version 20060415). The pipeline was optimized using the same parameters derived for the ultra-deep GOODS-North photometry data (Frayer et al. 2006b). We used a combination of the column median filter and a high-pass median time filter per detector to remove the instrumental artifacts ("streaking"). The filtering was done in two passes to remove the filtering artifacts ("negative side-lobes") around the brightest sources (Frayer et al. 2006a). A calibration factor of $702 \mathrm{MJy} \mathrm{sr}^{-1}$ per MIPS-70-unit (Gordon et al. 2007) was assumed, and the data have been color corrected by dividing by 0.918 (Stansberry et al. 2007). This color-correction factor is for a constant $v S_{v}$ SED
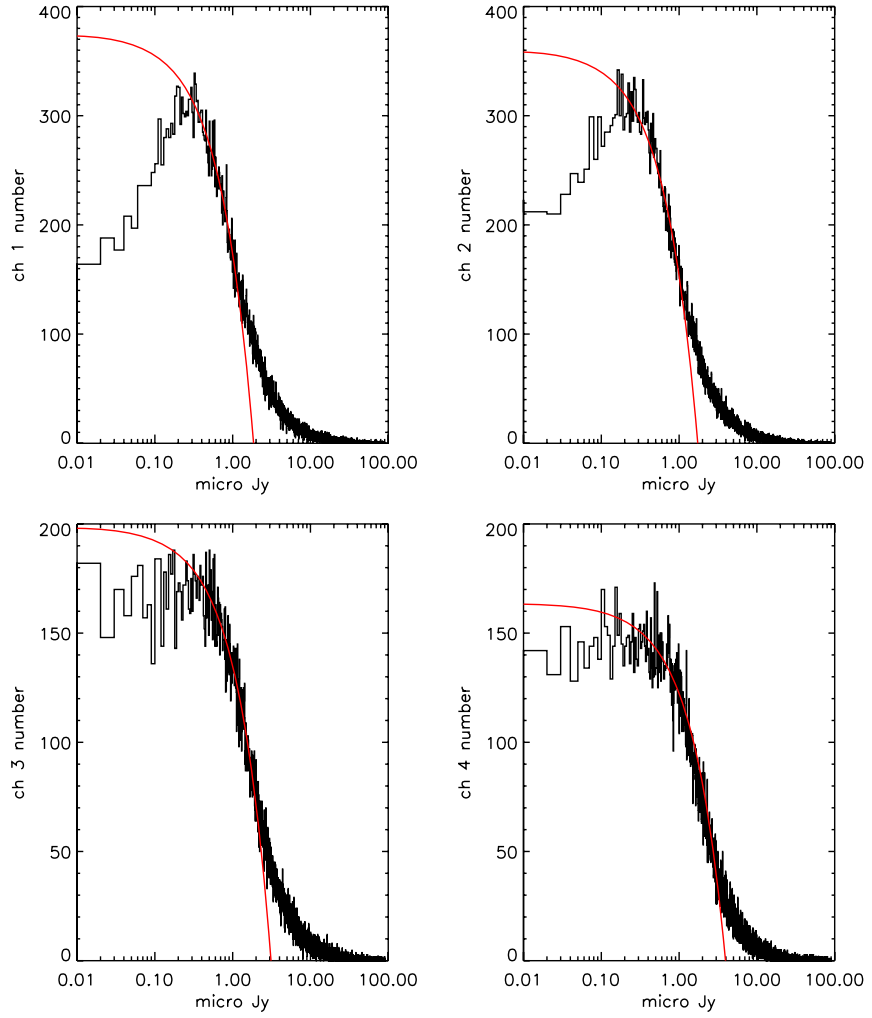

Figure 6. Number counts in the IRAC bands showing the completeness limits for each band. The histogram is the measured number of sources and the solid (red) line is a linear fit to the rising portion of the histogram. Completeness is determined where the measured number of sources drops below $95 \%$ of the expected number of sources based on the fits.

across the filter bandpass, but is appropriate to within $2 \%$ for a wide range of galaxy SEDs $\left(S_{v} \propto v^{-\alpha}, \alpha=0-3\right)$.

The data were combined using the SSC mosaicing software (MOPEX). PSF fitting has been found to be more accurate for MIPS $70 \mu \mathrm{m}$ data than aperture photometry (Gordon et al. 2007). Source extraction was done using the Astronomical Point-Source Extraction (APEX) tools within MOPEX. The $70 \mu \mathrm{m}$ peaks were fitted using the point response function (PRF; FWHM $=18^{\prime \prime}$.6) image derived from the extragalactic First Look Survey (Frayer et al. 2006a) because there are not enough isolated bright sources in the field to accurately derive our own PRF. Sixty-one sources were extracted with a signal-to-noise ratio $(\mathrm{S} / \mathrm{N})$ greater than $4(4.3 \mathrm{mJy})$. To avoid spurious sources at $70 \mu \mathrm{m}$ only those 42 sources with $24 \mu \mathrm{m}$ counterparts were included in this study. We do not expect any sources to be detected at $70 \mu \mathrm{m}$ that are not also detected at $24 \mu \mathrm{m}$ which we confirm by eye. The remaining unmatched sources are blends that we are unable to separate or noise peaks. We applied an aperture correction of 1.15 for emission outside of the adopted PRF (Frayer et al. 2009).

\subsubsection{MIPS $24 \mu \mathrm{m}$}

The $24 \mu \mathrm{m}$ data were taken with MIPS on Spitzer with a total exposure time of $1 \mathrm{hr}$ per pixel covering $225 \mathrm{arcmin}^{2}$ to a limiting $3 \sigma$ depth of $17.3 \mu \mathrm{Jy}$ (Program ID 1868). The data were taken in large-field photometry mode with a 30 s exposure time. A $3 \times 3$ MIPS field-of-view grid was mapped and repeated five times, with multiple dithers and chops. There were a total of 1080 separate exposures with a final total depth of 60 minutes per pointing on the sky. The MIPS data were processed by the 
Spitzer Science Center into individual image BCDs (pipeline version S12.4). However, substantial "jailbar" artifacts, as well as a significant gradient, were visible. All of the individual pointings were forced to a common background by applying an additive constant to the entire frame. A "delta-dark" was then generated from the median of all frames; the great degree of dithering in the data allows this process to reject all actual celestial objects in the frames from the median stack. That stack was then adjusted to a median overall zero value, and then subtracted from all the data. It currently is not known whether the gradient effect is additive or multiplicative, although our experience with other Si:As arrays of this kind strongly suggests (from a physical basis) that it is additive. However, we reduced the data both ways, and found no difference at any detectable level. The data were then co-added using the MOPEX software package onto exactly the same projection system as used for IRAC, albeit with $1^{\prime \prime} 2$ pixels.

IRAF DAOFIND was used for object detection. We supply the code with the PSF FWHM (5".8) and background sigma values taken by examining the image. DAOFIND then counts the flux within an annulus of diameter FWHM and flags any set of pixels as a detection where that flux is above a threshold of $5 \sigma$. To deal with confused sources, we perform object detection iteratively. After the first run through DAOFIND, all objects are subtracted from the image using a PSF determined from the detected objects. DAOFIND is then re-run on the residual image. Due to the large PSF and the lack of nearby galaxies in the field, with the exception of only a handful of galaxies, all MIPS detections appear as point sources. Photometry on all detected sources is done with the IRAF task ALLSTAR which fits PSF's to groups of objects simultaneously. An aperture correction of 1.4 is applied for flux beyond the 6.5 pixel radius at which the PSF star was normalized. This correction factor is calculated from a curve of growth based on the composite PSF star. Using this method the $3 \sigma$ detection limit is $17.3 \mu \mathrm{Jy}$. These noise properties are comparable to the GOODS slightly longer exposure (77 minutes) data set that has a $3 \sigma$ limit of $12 \mu \mathrm{Jy}$.

\subsection{Akari Space Telescope}

The $4,11,15$, and $18 \mu \mathrm{m}$ data were taken with the Infrared Camera (IRC; Onaka et al. 2007) on the Akari Space Telescope (Murakami et al. 2007) with a total exposure time of 1-2.5 hr per band per pixel covering $100 \operatorname{arcmin}^{2}$ to a $3 \sigma$ depth of 1.5 , 15,33 , and $45 \mu \mathrm{Jy}$, respectively. The details of this data set will be provided in E. Egami et al. (2009, in preparation), so here we only describe some basic data parameters.

The IRC was used to obtain images at 11,15 , and $18 \mu \mathrm{m}$ during 2006 October. These three Akari/IRC bands bridge the Spitzer imaging wavelength gap between IRAC $8 \mu \mathrm{m}$ and MIPS $24 \mu \mathrm{m}$. A $4 \mu \mathrm{m}$ image was also obtained since the 4 and $11 \mu \mathrm{m}$ data were taken simultaneously for the same region by design. Each image covers a field of view of $\sim 10^{\prime} \times 10^{\prime}$ (i.e., four times larger than that of IRAC) with a pixel scale of $2^{\prime \prime} .3-2$ '.5. The field center coordinates are 17:40:00 +69:00:00 (J2000).

Low-resolution infrared spectra were also taken for sources in the same $10^{\prime} \times 10^{\prime}$ area using the slit-less multi-object spectroscopy (MOS) mode of IRC (Ohyama et al. 2007). When combined, obtained spectra cover a wavelength range of 1.8$26.5 \mu \mathrm{m}^{8}$ with resolving powers of $20-50$. The integration times were several hours.

\footnotetext{
8 There is a gap in coverage at $13.4-17.5 \mu \mathrm{m}$ because the grism covering this range has been damaged.
}

\subsection{Palomar and KPNO Near-IR}

The $\mathrm{J}, \mathrm{H}$, and $\mathrm{K}_{s}$ observations were taken with the WIRC camera (Wilson et al. 2003) on the Palomar 200 inch Hale telescope and the FLAMINGOS camera on the KPNO $4 \mathrm{~m}$ telescope. Total exposure times at Palomar were 0.8, 0.7, and $1.6 \mathrm{hr}$ per pixel covering 120,120 , and $210 \operatorname{arcmin}^{2}$ to $3 \sigma$ limiting depths of $21.8,22.1$, and $21.2 \mathrm{mag}(\mathrm{AB})$ in $J, H$, and $K_{s}$, respectively. KPNO was used solely for the $J$ band with a total exposure time of $0.5 \mathrm{hr}$ per pixel covering $220 \mathrm{arcmin}^{2}$ to a $3 \sigma$ limiting depth of 21.4 mag (AB). Palomar data were taken on 2006 May 5, 2008 May 15-18, and 2008 June 27-28 and the KPNO data were taken on 2006 August 29-30. In both cases, the large format cameras are well matched to the field size allowing efficient observing.

The Palomar and KPNO data were reduced using a custom IDL pipeline (available from the authors). Blank sky images are generated from the actual survey data to serve as a temporally windowed measure of the dark current and sky. An initial pass through the data generates a first-order image reduction on which SExtractor is used to detect and mask objects and bad pixels within the data. A second pass utilizes the generated masks to reject pixels from the stacking process that creates the blank sky images. These "skydarks" were then subtracted from the data. Additional tasks repair other image defects, such as quadrant-level floating bias uncertainties. The data were flat fielded using dome flats. Each frame was individually calibrated both photometrically and astrometrically to 2MASS, which compensates for drifts in calibration due to non-photometric conditions. Individual images were re-projected using SWARP ${ }^{9}$ onto a common projection center used for all the other data sets. The data were then co-added using IRAF/IMCOMBINE, with relative weighting of the images based on their noise properties. The photometric and astrometric calibration of the final coadded mosaic was then further refined by a final rematch to the 2MASS survey. An object catalog was extracted using SExtractor, with a detection threshold of $2 \sigma$. The SExtractor MAG_AUTO photometric magnitude was used. Seeing was typically $1.1-1.15$. In general, the WIRC data are used for the survey as that data set is deeper than the FLAMINGOS data set. WIRC $3 \sigma$ detection limits vary from the center $(22.5,23.1$, $22.5)$ to the edge $(21.8,22.1,21.2)$ in $J, H, K(\mathrm{AB})$, respectively. The FLAMINGOS $3 \sigma$ detection limit is 21.4 mag (AB) in the $J$ band.

\subsection{MMT Near-IR}

The $J$ - and $H$-band observations were taken with the SAO Widefield InfraRed Camera (SWIRC) (Brown et al. 2008) at the $6.5 \mathrm{~m}$ MMT with total exposure times of 7.1 and $2.1 \mathrm{hr}$ covering 25 and $225 \operatorname{arcmin}^{2}$ to $3 \sigma$ limiting depths of 24.4 and 23.0, respectively. The data were taken over the course of two observing runs. The first set of observations was taken during the four nights of 2005 April 19-22. Conditions were not photometric, and the field was observed through thin cirrus. Total integration times were 14 minutes at both $J$ - and $H$ bands at each of nine positions arranged in an overlapping $3 \times 3$ configuration of a $5.1 \times 5.1$ field of view to cover roughly the central $15^{\prime} \times 15^{\prime}$ of the IRAC dark field. The total integration time was built up out of short, dithered $60 \mathrm{~s}(J)$ and $30 \mathrm{~s}(H)$ exposures. The seeing was variable but remained below 1" FWHM for most of the run. The second set of observations was carried out during

\footnotetext{
9 http://terapix.iap.fr/rubrique.php?id_rubrique $=49$
} 
the three nights of 2006 May 12-14. During this campaign only the central $5^{\prime} \times 5^{\prime}$ of the IRAC cal field (the subregion with the deepest overall IRAC integration), was observed, and only at the Jband. A total of $25770 \mathrm{~s}$ dithered exposures were acquired through variable cirrus with sub-arcsecond seeing for a total combined exposure time of $5 \mathrm{hr}$.

The individual exposures were processed using standard methods. All frames were dark- and bias-subtracted using dark frames taken at the beginnings and ends of the corresponding nights. A custom mask was constructed using a median stack of all the $60 \mathrm{~s} J$-band exposures from the 2005 run, so as to exclude large regions of bad and noisy pixels from subsequent analysis. The celestial coordinates, which were initially only accurate to within $20^{\prime \prime}$, were refined and verified with the wcstools package by reference to bright 2MASS stars. The images were flattened with skyflats. This was an iterative process whereby a crude, initial mosaic was first constructed to reveal faint objects not easily detected in the individual exposures. Objects in this initial mosaic were then identified and masked, and the deeper object masks were subsequently translated to the coordinate frames of all individual frames. In this way, with the much deeper object masks, much-improved skyflats were created from the stack of individual frames. Specifically, this technique eliminated the negative artifacts that arose from repeated placement of faint-but-significant objects on the same parts of the detector array by the dither pattern.

Because none of the data were taken on photometric nights, the photometric calibrations were referenced to an ensemble of well-detected 2MASS stars. Final mosaics were constructed using 0 '. 15 pixels by co-adding the individual frames using sigma clipping to eliminate cosmic rays (CRs). Two wide, shallow $15^{\prime} \times 15^{\prime}$ mosaics were made having $3 \sigma$ depths of 22.7 $(J)$ and $23.0(H)$ AB magnitudes, based on the noise measured within a $3^{\prime \prime}$ diameter aperture. In the smaller but deeper mosaic from 2006 a $3 \sigma$ depth of 24.4 AB magnitude was estimated within the same aperture. The FWHM in the final mosaics was roughly 0.75 .

Photometry was measured in the final mosaics with SExtractor, using MAG_AUTO as a measure of total magnitude, thus not requiring an aperture correction. For the wide-field mosaics, SExtractor was used in two-image mode. In this case, the IRAC $3.6 \mu \mathrm{m}$ mosaic was used as the detection image, and the $J$ - and $H$-band mosaics were used as the measurement images. For the deep $J$-band mosaic, SExtractor was used in single-image mode. Given the $0{ }^{\prime \prime} 15$ pixels and the 0.75 seeing, six connected pixels each individually detected at the $1.5 \sigma$ level were required for a source detection. The resulting deep $J$-band catalog contains over 13,000 sources.

\subsection{MMT Optical}

The $z^{\prime}$ observations were taken with the Megacam camera at the $6.5 \mathrm{~m}$ MMT with a total exposure time of $3.6 \mathrm{hr}$ per pixel covering $580 \mathrm{arcmin}^{2}$ to a $3 \sigma$ limiting depth of 25.9 mag (AB). The observations consisted of a series of $500 \mathrm{~s}$ exposures taken on the nights of 2007 June 1-4 under photometric conditions, except for the night of 2007 June 1, when some thin cirrus was present. Seeing varied from as low as 0.6 to as high as $11^{\prime \prime} .7$, and averaged about 1 1.0. The data were reduced interactively using standard techniques on a dedicated pipeline machine hosted at SAO. Bias-subtracted and flattened exposures were treated to remove CRs and bad pixels before calculating the coordinates using stars from the USNOB1.0 catalog and correcting the photometry for off-axis scattered light. The resulting exposures were spatially registered to a common coordinate system. All frames taken on the night of 2007 June 1 were then flux calibrated using exposures from the photometric nights. All the frames were then scaled appropriately and co-added to create the final $z^{\prime}$ mosaic. The mosaic covers roughly $24^{\prime} \times 24^{\prime}$ covering the entire IRAC survey field where the center has the deepest exposure time of $3.6 \mathrm{hr}$ tapering toward the field edge.

The $z^{\prime}$ mosaic was photometered with SExtractor in singleimage mode, using MAG-AUTO as a total magnitude. Because the seeing was measured to be approximately $1^{\prime \prime} .0$ in the combined image, the detection algorithm was configured to identify all objects having at least 12 connected 0 '. 32 pixels lying $1 \sigma$ above the background. Using the coverage map, portions of the mosaic observed three or fewer times were masked, so that photometry was measured only for sources observed at least four times by Megacam. We estimate that the depth reached is 25.9 AB magnitude, $3 \sigma$, within a $2^{\prime \prime}$ aperture.

\subsection{HST/ACS Optical}

HST/ACS F814W observations were taken with a total exposure time of $1.4 \mathrm{hr}$ per pixel covering $260 \mathrm{arcmin}^{2}$ to a $3 \sigma$ limiting depth of $28.6 \mathrm{mag}$ (AB) (Program ID 10521). ACS WFC is a mosaic of two $2048 \times 4096$ SITE CCDs with a pixel scale of 0. . $05 /$ pixel ( $15 \mu$ pixels), so that the full field of view per exposure is $11.3 \mathrm{arcmin}^{2}$. Observations were made over 50 orbits between 2006 November 28 and 2006 December 07 using SIDE-2 electronics. The observations were divided into 25 pointings, and each pointing was in turn divided into eight roughly 10 minute exposures for a total of 200 exposures. We used a small dither between exposures at each pointing for CR rejection and to cover the gap between the two ACS CCDs (2".5). We also employ a $\sim 0.5$ overlap between pointings. The entire ACS mosaic is contained within the Spitzer IRAC map.

The ACS pipeline calacs was used for basic reduction of the images including overscan and bias subtraction, CR rejection, dark subtraction, and flat fielding. Beyond this base reduction we developed our own method of further reducing and mosaicing the 25 pointings. The three largest challenges in doing this were the large amount of memory required to combine $200160 \mathrm{Mb}$ fits files, getting the astrometry correct given distortions and relative shifts between images caused by thermal effects in the telescope, and the variation in background level due to the four amplifiers used to readout the two CCDs. MULTIDRIZZLE alone is unable to account for these effects. Our pipeline uses a combination of the IRAF tasks MULTIDRIZZLE, TWEAKSHIFTS, and CCMAP along with automatic background subtraction by quadrant and SWARP for the final mosaicing. The final combined mosaic image is 1.7 GB in size and covers a $\sim 20^{\prime}$ diameter rough circle in the center of the IRAC dark field with the native 0.05 per pixel resolution and $0^{\prime \prime} 1$ seeing.

The photometry zero point was taken from the headers. SExtractor was used with the same basic setup as the Palomar optical data for source detection and extraction (see Section 2.7). The SExtractor MAG_AUTO is used in the catalog which uses a Kron total magnitude when there are no near neighbors to bias the measurement and a corrected isophotal magnitude otherwise. The $3 \sigma$ detection limit for point sources is $I=$ $28.6(\mathrm{AB})$. There are $\sim 51,000$ sources in the catalog with detections in both ACS and IRAC.

\subsection{Palomar Optical}

The $u^{\prime}, g^{\prime}, r^{\prime}$, and $i^{\prime}$ data were taken with the Large Format Camera at the 200" Hale telescope at Palomar with total 
exposure times of $6.0,6.2,4.1$, and $4.7 \mathrm{hr}$ per pixel covering $450 \mathrm{arcmin}^{2}$ to $3 \sigma$ limiting depths of $27.2,27.1,26.6$, and 26.0 mag (AB), respectively. Observing runs occurred on 2004 July 22-24 and 2004 August 8-10. All observing nights had less than $50 \%$ Moon illumination. The LFC is a mosaic of six $2048 \times 4096$ CCDs with a pixel scale of 0 '. $18 /$ pixel $(15 \mu$ pixels $)$, so that the full field of view per exposure is roughly a $24^{\prime}$ diameter circle. Images were randomly dithered between exposures to account for the gap between chips $\left(15^{\prime \prime}\right)$. Integration times were typically $360 \mathrm{~s}$ in $r^{\prime}$ and $i^{\prime}, 900 \mathrm{~s}$ in $u^{\prime}$, and $600 \mathrm{~s}$ in $g^{\prime} . u^{\prime}$-band science and calibration data were binned $2 \times 2$ pixels.

Reduction of this Palomar data follows standard procedures. The images were cross-talk and overscan corrected using IRAF tasks. A combined bias frame was then made from roughly five overscan-corrected individual bias frames and applied to the data to remove the large-scale bias. No dark current correction was required. Pixel-to-pixel sensitivity variations were corrected in all images using 15 median-combined dome flats taken throughout the run with 7000-9000 counts each. Although twilight flats were taken during the August run, they were not required in the reduction. $i^{\prime}$-band images were fringe corrected using a median smoothed, object removed, bad-pixel masked combination of all science frames.

The IRAF tasks MSCGETCATALOG, MSCCMATCH, and MSCCSETWCS were used to find and apply $x$ - and $y$-shifts and rotations to each frame by tying it to the USNO-A2 positions. Fits were done manually by ruthlessly rejecting outliers while maintaining even coverage over the field. A combined bad pixel mask was made for all CRs, hot pixels, and satellite trails for each image using CRAVERAGE and masking by hand. The bad pixel mask was used with the IRAF task FIXPIX which does a linear interpolation over the effected pixels. To remove gradients in the background we use MSCSKYSUB to fit a second-order Legendre to the background of each image and subtract it. SWARP was then used to re-project and combine together all images in each filter.

Photometric calibration was performed in the usual manner using a field of Sloan Digital Sky Survey (SDSS) well measured stars at a range of airmasses. Photometric nights were analyzed together; solutions were found in each filter for a common magnitude zero point. All nights except August 10 were photometric. Those exposures taken in non-photometric conditions were individually tied to the photometric data using roughly 10 stars well distributed around each frame.

We use SExtractor to both find all objects in the combined frames, and to determine their shape parameters. The detection threshold was defined such that objects have a minimum of six contiguous pixels, each of which are greater than $1.5 \sigma$ above the background sky level. We choose these parameters as a compromise between detecting faint objects in high signal-tonoise regions and rejecting noise fluctuations in low signal-tonoise regions. This corresponds to $3 \sigma$ point source detection limits of 27.2, 27.1, 26.6, and $26.0 \mathrm{mag}(\mathrm{AB})$ in $u^{\prime}, g^{\prime}, r^{\prime}$, and $i^{\prime}$, respectively. Shape parameters are determined in SExtractor using only those pixels above the detection threshold.

When making the catalog, we use an aperture magnitude for objects fainter than 22.5 and a Kron magnitude from SExtractor for all objects brighter than 22.5. We choose a kron factor of 2.5 and a minimum radius of 3.0 pixels as can be shown to give the closest estimate to total magnitudes (Bertin \& Arnouts 1996). The fainter objects are all point sources and so do not benefit from shape information in their photometry. Aperture sizes are 3.' $6,5^{\prime \prime} .6,4^{\prime \prime} .1$, and $4^{\prime \prime} .5$ for $u^{\prime}, g^{\prime}, r^{\prime}$, and $i^{\prime}$, respectively. Aperture sizes were chosen based on a curve of growth of a bright star in the field. We choose large apertures $(\sim 4 \times$ FWHM $)$ to enclose "all" of the flux and therefore not require an aperture correction.

\subsection{Palomar Optical Spectroscopy}

Spectroscopy was done with the COSMIC spectrograph at the Palomar Hale 200" telescope. COSMIC, at prime focus, has a 13.6 field of view, and 0.'4 pixels. Observations were made over four nights in 2007 and 2008 June with the $3001 \mathrm{~mm}^{-1}$ grating with a dispersion of $3 \AA$ per pixel. We chose a slit width of $1^{\prime \prime} .5$ to match our $1-1^{\prime \prime} .5$ seeing. The optical band covered by this instrument, roughly 3500 to $9000 \AA$, includes such spectral features as $\mathrm{CaH} \& \mathrm{~K}$, [O II], [O III], $\mathrm{H} \alpha, \mathrm{H} \beta, \mathrm{H} \delta, G$ band, and the $4000 \AA$ break. During both runs we were able to observe a total of 11 slitmasks of $\sim 25$ galaxies each with exposure times of on average 80 minutes divided into multiple exposures. One $\mathrm{HgAr}$ lamp and one flat exposure were taken through each mask at the beginning of the night for calibration. Targets were selected to be galaxies (non-stellar PSFs) brighter than $r=21$ (AB) with priority in mask design given to those with MIPS 24 or $70 \mu \mathrm{m}$ detections to boost the chance of seeing an emission line and thereby getting a secure redshift.

Reduction was done with IRAF mainly through the Bogus2006 ${ }^{10}$ scripts. Prior to running BOGUs, images were overscan and bias subtracted. Bogus itself does a two-dimensional reduction including flat fielding, CR removal, sky subtraction, fringe suppression, and combination of frames. The same reduction is performed on both science images and arcs. The standard IRAF tasks of APALL, IDENTIFY, and DISPCOR were used to wavelength correct, trace, and extract the spectra with a secondary background subtraction for minor level changes. Onedimensional spectra were extracted for a total of 200 galaxies with measurable continuum. Although spectroscopic standards were observed during each run, we did not flux calibrate the spectra as we are only interested in determining redshifts (see Section 4).

\subsection{Chandra ACIS-I}

Chandra ACIS-I observations were taken with a total exposure time of $28 \mathrm{hr}$ per pixel covering $290 \mathrm{arcmin}^{2}$ to a limiting depth of $3.6 \times 10^{-16} \mathrm{ergs} \mathrm{s}^{-1} \mathrm{~cm}^{-2}$. The camera is a $2 \times 2$ mosaic of four $1024 \times 1024$ frontside-illuminated CCDs arranged to be tangent to the mirror assembly in the focal plane. The pixel scale is 0 ". 49/pixel (24 $\mu \mathrm{m}$ pixels), so that the full field of view per exposure is roughly $290 \mathrm{arcmin}^{2}$. Observing was done in three separate observations at different pointing angles to reduce the effect of the gap between chips (11"); $50 \mathrm{ks}$ on 2007 July 23, $22 \mathrm{ks}$ on 2007 September 28, and $28 \mathrm{ks}$ on 2007 September 29, for a total exposure time of $100 \mathrm{ks}$ (observation IDs 7359, 8471, 9595). Observations were done in timed exposure mode (TE) with very faint format (VFAINT) in order to measure events in $5 \times 5$ pixel islands instead of smaller regions which has the advantage of better determination of background events such as CRs. We know from the ROSAT survey that there are no bright objects in the field such that pile-up of flux would be a problem in these larger event regions. The data were pre-processed by the Chandra X-ray center with standard data processing (SDP) version 7.6.11.1. Further reduction was done with the Chandra Interactive Analysis of Observations software (CIAO 4.0). This version of the SDP includes identifying and correcting for hot

\footnotetext{
${ }_{10}$ https://zwolfkinder.jpl.nasa.gov/ stern/homepage/bogus.html
} 
pixels and CR afterglows (residual charge on frames after a CR hit).

In creating an event list we include background cleaning with the code ACIS_PROCESS_EVENTS which flags possible background events based on counts in the outer ring of the $5 \times 5$ event island so that they can be removed in the filtering phase. We then filter the event list for the number of pixels above a threshold and flags for bad pixels or CRs. Second, frames which are not considered good science frames due either to the telescope motion, angle, or temperature are removed from the event list. Lastly, we filter the data on energy range, keeping only energies between 0.3 and $7 \mathrm{KeV}$ where the particle background is fairly flat and quantum efficiency is the highest.

Because the observations were taken in three different exposures we correct the astrometry before combining individual observations into an exposure map and fluxed image. To do this we use a preliminary run of WAVDETECT to make a source list and then REPROJECT_ASPECT to reproject the images to a reference image (in this case the first, longest observation ID 7359). MERGE_ALL is used to create the final combined image and exposure map. After combination we examine the data for background flares and remove anomalous high background times from the image. We perform source detection and photometry on the full $0.3-7 \mathrm{Kev}$ band data using WAVDETECT which operates by correlating the data with "mexican hat" wavelet functions on size scales from 1 to 8 pixels. The significance threshold parameter in WAVDETECT is set such that we expect approximately one false detection per $1000 \times 1000$ pixel area, or approximately four in our field. There are 121 total source detections.

Photometry with WAVDETECT sums the total counts within the source cell defined by the shape which encompasses all positive count pixels around the source detection. Backgrounds are determined in the wavelet analysis. To convert from count rate to flux units we assume an average spectral shape of $\Gamma=1.4$ (Giacconi et al. 2002; Elvis et al. 2009; Laird et al. 2009). Along with the local galactic NH value of $4 \times 10^{20} \mathrm{~cm}^{-2}$ this gives us energy conversion factors of $5.49 \times 10^{-12}, 1.8 \times 10^{-11}$, and $1.08 \times 10^{-11}$ in the soft, hard, and total bands, respectively. Using $\Gamma=1.7$ (Manners et al. 2003) changes these conversion factors by $\sim 10 \%$. The minimum number of counts we have detected in the full band is $3.3 \pm 2.0$, corresponding to $3.6 \pm 2.2 \times$ $10^{-16} \mathrm{erg} \mathrm{cm}^{-2} \mathrm{~s}^{-1}$.

\section{THE CATALOG}

The catalog is built by matching sources from each band to the remainder of the catalog within a radius that is similar to that band's PSF size. The disadvantages of this method are missmatches due to noise detections and deblending imperfections. A noise detection specifically in the ACS band will propagate through to the IRAC bands because of our photometry methods (see Section 2.1.1). Saturated stars with diffraction spikes often produce spurious signals. Fortunately, this field has few/no bright stars by design (see Section 1). The brightest star in the field is $12.2 \mathrm{mag}(\mathrm{AB})$ in $\mathrm{F} 814 \mathrm{~W}$. The main source of error in the matching will be incorrect matches because of multiple sources within the match radius. For this reason we match catalogs with similar PSF sizes. For those bands with especially large PSFs we are careful to match sources with other large PSF bands, and not directly to our smallest PSF catalog. Specifically the MIPS $24 \mu \mathrm{m}$ data set is matched to the IRAC source list, as the catalog nearest in PSF size to itself. Second, the MIPS $70 \mu \mathrm{m}$ data set is matched to the MIPS $24 \mu \mathrm{m}$ data. This method means that we will lose any MIPS $24 \mu \mathrm{m}$ sources which are not detected at IRAC wavelengths and similarly any $70 \mu \mathrm{m}$ sources which are not detected at $24 \mu \mathrm{m}$. We expect these objects to be extremely rare, if they exist at all, and therefore they will be searched for in a different manner.

For this catalog we do not use matched apertures, but instead we calculate a "total" flux in each band. We do not want to lose information by smoothing all the bands to the same resolution, but instead keep all information possible at each wavelength. To this end, we use aperture corrections where necessary, as discussed above.

By far, the HST ACS F814W catalog has the most number of sources and so dominates the spatial distribution of sources. As mentioned in Section 2.1.1, the ACS catalog is used as the detection image for the IRAC data. Other optical and near-IR bands are matched to that merged catalog of ACS and IRAC. In total, there are 86,815 sources in the matched catalog. This catalog will be made publicly available at a future date. At this time all of the space-based data are available from their respective archives.

As a check of the catalog photometry, we present color-color diagrams of stars in the field. Stars are a good population on which to test our photometry since they follow well-defined tracks in color space. We select stars from our ACS catalog. A plot of the SExtractor ISOAREA parameter as a function of magnitude clearly separates a ridge of unresolved point sources from the resolved sources because unresolved sources have smaller ISOAREA than galaxies at any given magnitude. Although we have used our highest resolution data to pick out the stars, there are undoubtedly interlopers from higher redshift compact galaxies or quasars. In total, we have $\sim 150$ point sources with detections in $u^{\prime}, g^{\prime}, r^{\prime}, i^{\prime}, J$, and $H$. In Figure 7 , we compare the optical and near-IR colors of these stars to those from synthetic stellar spectra from the BaSeL library (Lejeune \& Schaerer 2001). The comparison sample includes synthetic stars with masses from $0.4-0.8$ to $120-150 M_{\odot}$ and metallicities $Z=0.0005-0.1$ measured at ages between $1000 \mathrm{yr}$ and 1620 Gyr. We find good agreement between the real star colors and those from the theoretical models.

\section{PHOTOMETRIC REDSHIFTS}

The combined IRAC and ACS catalog contains over 50,000 objects which makes acquisition of spectroscopic redshifts impractical. Even confirmation spectroscopy of red galaxies at $z=1$ in our three candidate clusters will require many nights on 8-10 m class telescopes and is therefore also impractical. In lieu of spectroscopy we use our extensive multi-wavelength, broadband catalog to build SEDs using up to 13 bands $\left(u^{\prime}, g^{\prime}\right.$, $\left.r^{\prime}, i^{\prime}, \mathrm{F} 814 \mathrm{~W}, z^{\prime}, J, H, K, 3.6,4.5,5.8,8.0 \mu \mathrm{m}\right)$ from which we derive photometric redshifts.

These SEDs are fitted with template spectra from Polletta et al. (2007). ${ }^{11}$ These templates have been used successfully by a number of surveys at a range of redshifts for all galaxy types (Adami et al. 2008; Negrello et al. 2009; Salvato et al. 2009; Ilbert et al. 2009). Because the templates include the IR spectral region (empirically built from observations in SWIRE), we find them the best choice to use as models for this data set. We use 15 templates including ellipticals, spirals, star-forming galaxies, and AGNs. Photometric redshifts are calculated using HYPERZ; a chi-squared minimization fitting program including a correction for interstellar reddening (Bolzonella et al. 2000; Calzetti et al.

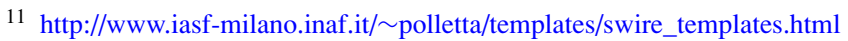



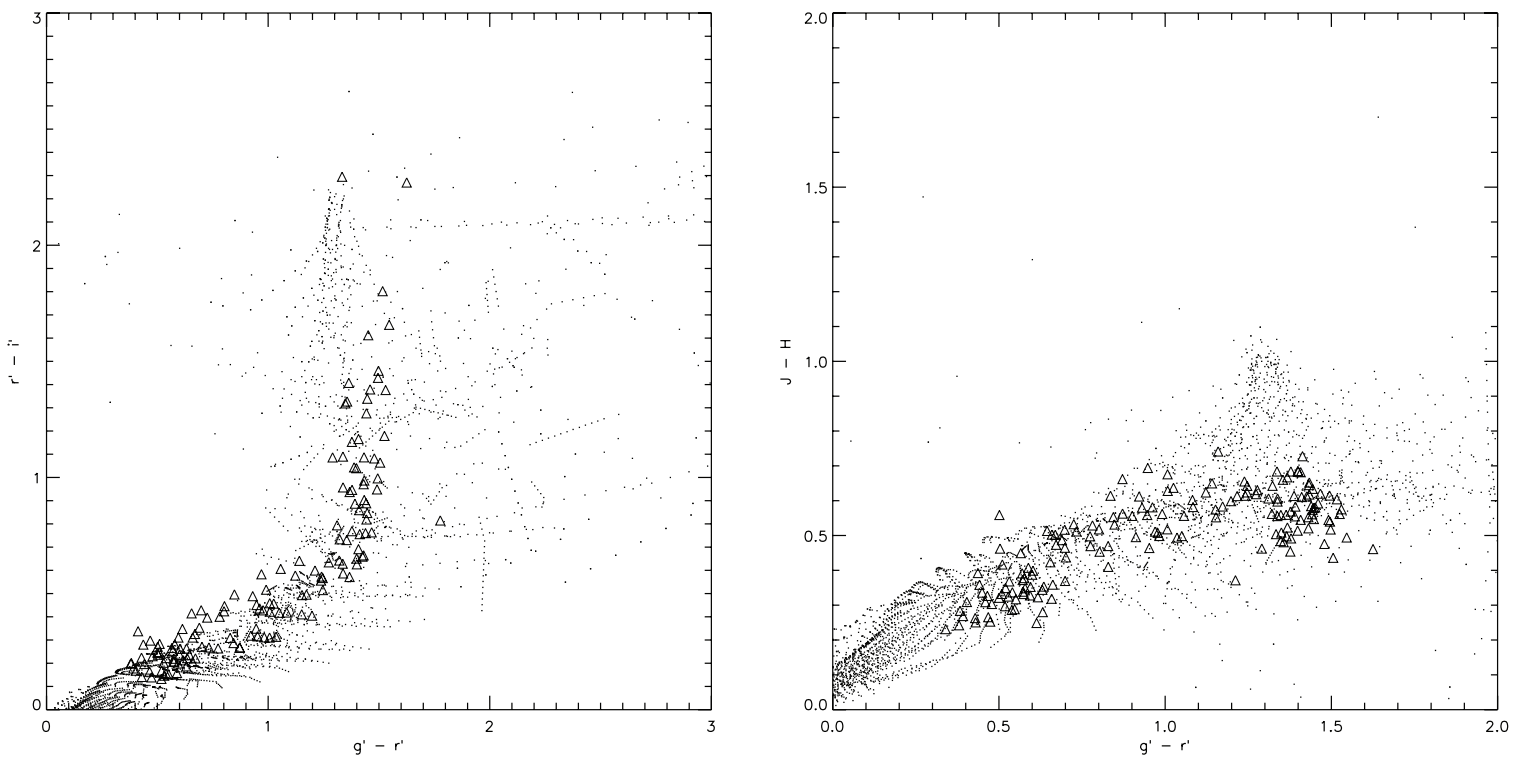

Figure 7. Optical and near-IR color-color diagrams as a check of photometry. Colors from synthetic stellar spectra from Lejeune \& Schaerer (2001) for a set of stars covering a range in mass and metallicity are shown as black points. The 150 stars from the dark field are shown as triangles.
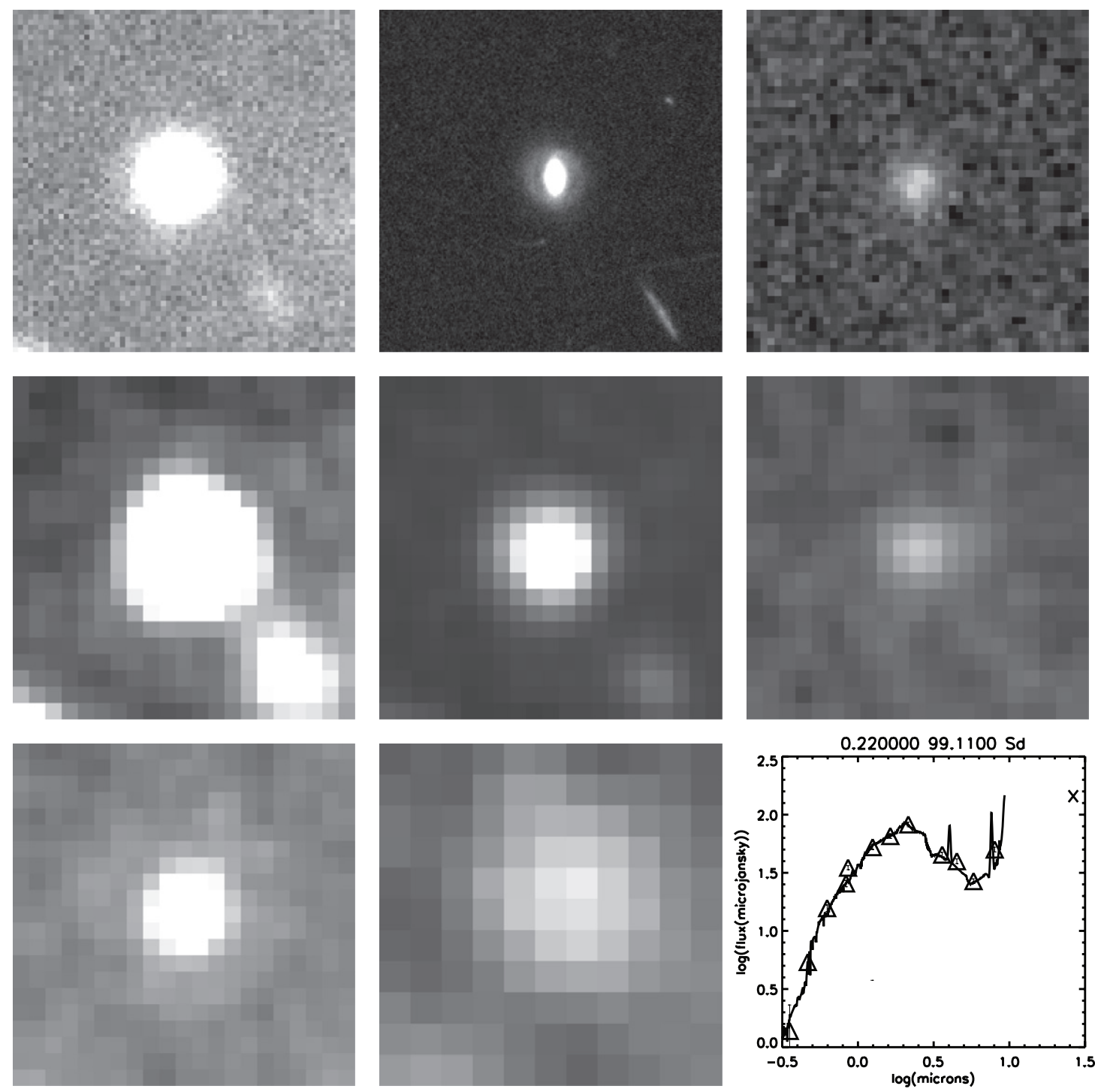

Figure 8. Example spiral galaxy with a well-fit photometric redshift by hyperz. The thumbnails show a $12^{\prime \prime} \times 12^{\prime \prime}$ box in $r^{\prime}, H S T / \mathrm{ACS}$ F814W, $K, 3.6,4.5,5.8,8.0$, $24 \mu \mathrm{m}$, and the fitted SED. The title of the SED plot gives the best-fit photometric redshift, the probability that redshift is good, and the name of the best-fit template. Triangles are data used in the fit, the line is the fit, and the cross is the $24 \mu \mathrm{m}$ data point, not used in the hyperz fit, but plotted here for completeness. 


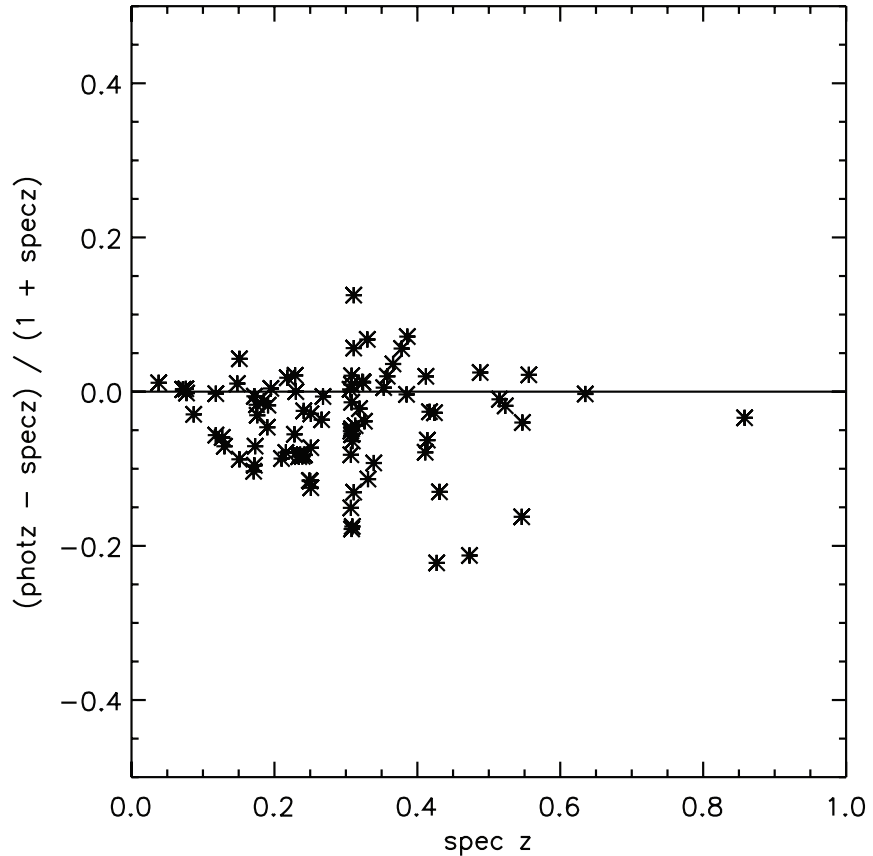

Figure 9. Comparison of spectroscopically and photometrically determined redshifts for 87 galaxies. The scatter implies an error on the photometric redshifts of $0.064(1+z)$.

2000). An example of a well-fit galaxy is shown in Figure 8. Errors in photometric redshifts are determined by comparing the photometric redshifts with spectroscopic redshifts.

Spectroscopic redshifts were determined using both IRAF tasks EMSAO and XCSAO. Specifically EMSAO searches the spectrum for both absorption and emission lines which it correlates with a given line list. XCSAO cross-correlates the spectrum with known galaxy templates which allows us to use features such as the $4000 \AA$ break and the rest of the spectral shape to identify redshifts. Both techniques were used together to arrive at the best-fit redshift for each galaxy. We used 17 spectral templates of galaxies and AGN from the compilation of the HST Calibration Database System (Francis et al. 1991; Kinney et al. 1996;
Calzetti et al. 1994). We do not use the SWIRE SED templates here (as we do for the photometric redshifts above) because the optical spectra in the SWIRE templates are based on modeling and their true strength lies in their IR treatment. Since our spectroscopy is in the optical, we use the Calibration Database System templates because they are based more reliably on UV and optical observations. We applied a very strict requirement that all emission and absorption features in the one-dimensional spectra were confirmed by eye on the two-dimensional spectra and that multiple lines be identified in all cases to avoid incorrect redshift determination due to $\mathrm{CRs}$ or noise features from sky line subtraction.

We were able to successfully determine redshifts for 87 galaxies. This represents a conservative sample of "good" redshift determinations defined to have either high signal-tonoise emission lines or multiple absorption lines and good cross correlations. We then compare the spectroscopic to photometric redshifts to quantify the error on the latter (Figure 9). There are cases where HYPERZ has failed to fit the correct redshift. In these galaxies, it appears that HYPERz has not chosen a template that goes through any of the photometric points, completely missing the SED shape. This could be due to a failure of hyperz or the errors in the photometry. Those galaxies, as characterized by a $\chi^{2}$ value greater than 50 , are not included in this comparison sample. The error on the photometric redshifts is $0.064(1+z)$. Note that this error is quoted as a function of redshift and so takes into account the increasing scatter with $z$. This accuracy is similar to other IRAC-based multi-wavelength studies (Brodwin et al. 2006; Rowan-Robinson et al. 2008).

\section{INITIAL SCIENCE RESULTS}

Given this unique data set, we are carrying out scientific programs to exploit the available information. Krick et al. (2008) have discovered a large-scale structure at redshift 1 consisting of three galaxy clusters. These clusters show evidence of a buildup of the red cluster sequence at redshift 1 . In a study of the star formation and AGN fraction in those clusters, Krick et al. (2009) have found evidence for star formation in star-forming galaxies to look the same regardless of environment, but a dense
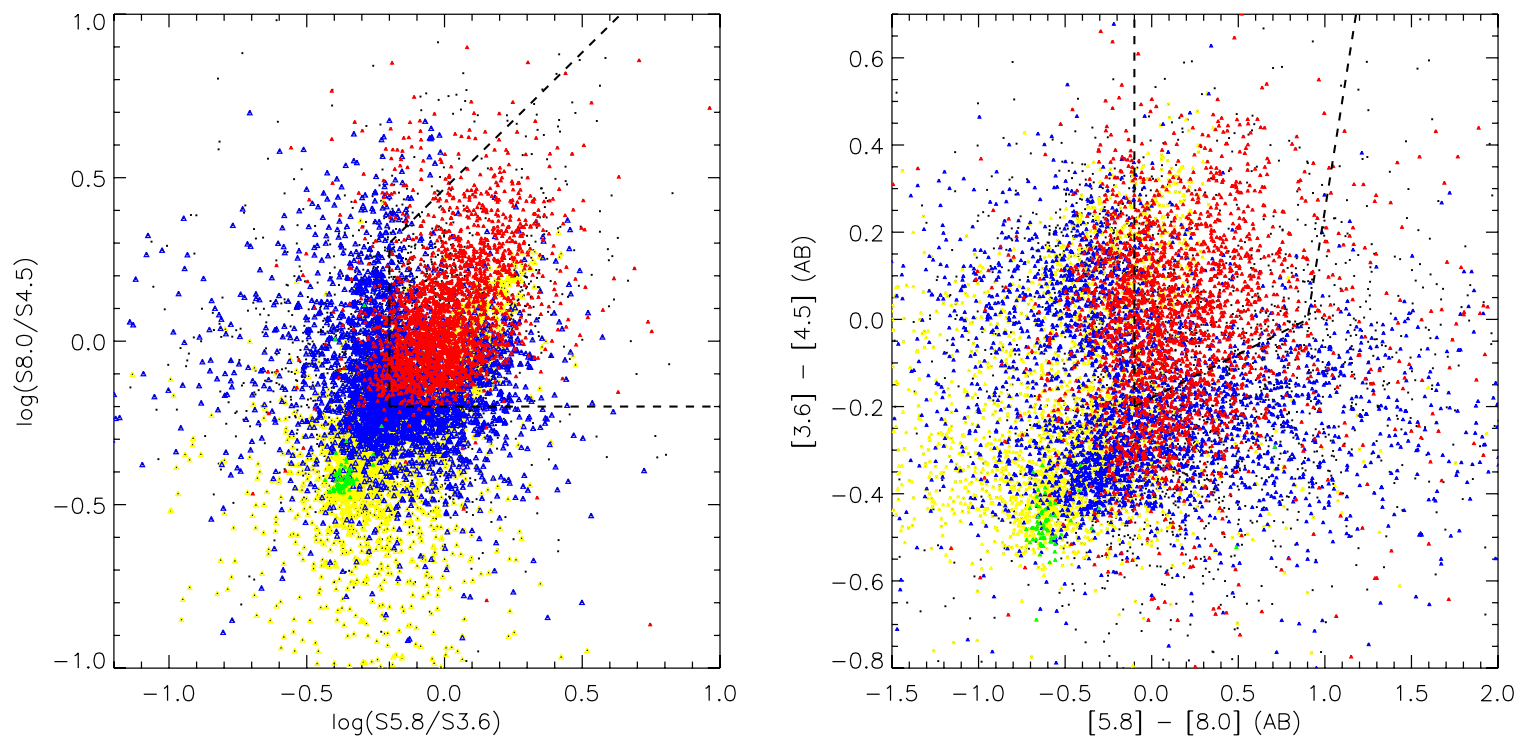

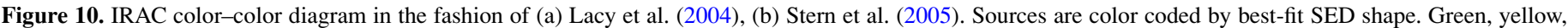

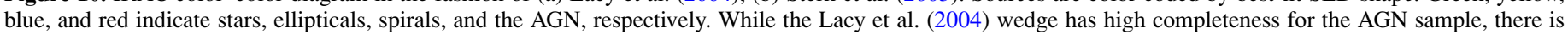
significant contamination, and the Stern et al. (2005) wedge shows lower completeness but less contamination. 
environment does strengthen the suppression of star formation and AGN activity in clusters over the field from redshift 1 to the present.

Ongoing work continues on finding and characterizing highredshift galaxies $(z>6)$, Compton thick AGNs, the lowest mass brown dwarfs, population III SNe (Frost et al. 2009), the reddest objects in the universe from their $24 \mu \mathrm{m}-r$ colors, and other interesting phenomena, the study of which is uniquely suited to this field. As this is the only mid-IR data set with time domain information on a 5+ year time line, we explore the variable and transient objects in the field, from stars to AGNs (Hund et al. 2006; Surace et al. 2007, and M. Foesneau et al. 2009, in preparation). One such application is to study the extragalactic variability in mid-IR to set constraints on the nature of dust around the AGN.

As a representation of the data, we include here a discussion of IRAC color-color diagrams to both differentiate between AGNs and star-forming galaxies (Lacy et al. 2004; Stern et al. 2005) and demonstrate the effectiveness of our ACS-based IRAC photometry. Figure 10 shows all sources originally detected in the four IRAC bands before adding ACS shape information. Here we have separated the sample based on SED classifications into a spiral, elliptical, AGN, and stellar sample. Those SEDs best fit with composite templates are not colored in the plot. Deep surveys such as the IRAC dark field, in opposition to the shallower, wide-area, surveys like the Spitzer first look survey and the IRAC shallow survey (Eisenhardt et al. 2004) show a larger amount of contamination in the AGN wedges from intermediate-redshift spiral and elliptical galaxies (Sajina et al. 2005; Barmby et al. 2006; Barger et al. 2008). Those AGNs which are blueward (left) of the Lacy et al. (2004) wedge are AGNs at redshift $\sim 1.0$ that have a stellar peak in addition to a power-law shape, and that stellar peak has been shifted into the IRAC $3.6 \mu \mathrm{m}$ band. We see from these plots that our SED fitting produces nice results for the morphologies of the galaxies.

Second, we discuss the effect of our ACS-based IRAC photometry on the scatter in the color-color diagrams. We do see larger scatter than the original Lacy et al. (2004) and Stern et al. (2005) diagrams because this is a deeper survey. The larger scatter is also seen in other deep surveys like that in the Extended Groth Strip (Barmby et al. 2006). We use this scatter in the colorcolor diagrams to quantify the difference between using the IRAC only photometry and the ACS-based IRAC photometry. We use the same sample of $\sim 11,000$ galaxies originally detected in all four IRAC bands to make color-color distributions of both IRAC only and ACS-based IRAC photometry. The change in scatter is quantified with the F-test where the ratio of variances gives probabilities that the scatters in the distributions are the same. Those probabilities are less than $1 \times 10^{-14}$ indicating that the scatter is statistically significantly reduced if we use the ACS-based photometry instead of the IRAC only photometry.

We thank the anonymous referee for useful suggestions on the manuscript. This research has made use of data from the Two Micron All Sky Survey, which is a joint project of the University of Massachusetts and the Infrared Processing and Analysis Center/California Institute of Technology, funded by the National Aeronautics and Space Administration and the National Science Foundation. This work was based on observations obtained with the Hale Telescope, Palomar Observatory as part of a continuing collaboration between the California Institute of Technology, NASA/JPL, and Cornell University, the Spitzer Space Telescope, which is operated by the Jet Propulsion Labo- ratory, California Institute of Technology under a contract with NASA, and the NASA/ESA Hubble Space Telescope, obtained at the Space Telescope Science Institute, which is operated by the Association of Universities for Research in Astronomy, Inc. Support for program \#10521 was provided by NASA through a grant from the Space Telescope Science Institute, which is operated by the Association of Universities for Research in Astronomy, Inc., under NASA contract NAS 5-26555. FLAMINGOS was designed and constructed by the IR instrumentation group (PI: R. Elston) at the University of Florida, Department of Astronomy, with support from NSF grant AST97-31180 and Kitt Peak National Observatory. Observations reported here were obtained at the MMT Observatory, a joint facility of the Smithsonian Institution and the University of Arizona. Support for this work was provided by the National Aeronautics and Space Administration through Chandra Award Number G07-8120 issued by the Chandra X-ray Observatory Center, which is operated by the Smithsonian Astrophysical Observatory for and on behalf of the National Aeronautics Space Administration under contract NAS8-03060.

Facilities: Hale (LFC, WIRC, COSMIC), MMT(Megacam, SWIRC), HST (ACS), Spitzer (IRAC, MIPS), Akari, CXO (ACIS), KPNO()

\section{REFERENCES}

Adami, C., Ilbert, O., Pelló, R., Cuillandre, J. C., Durret, F., Mazure, A., Picat, J. P., \& Ulmer, M. P. 2008, A\&A, 491, 681

Barger, A. J., Cowie, L. L., \& Wang, W.-H. 2008, ApJ, 689, 687

Barmby, P., et al. 2006, ApJ, 642, 126

Bertin, E., \& Arnouts, S. 1996, A\&AS, 117, 393

Bolzonella, M., Miralles, J.-M., \& Pelló, R. 2000, A\&A, 363, 476

Brodwin, M., et al. 2006, ApJ, 651, 791

Brown, W. R., McLeod, B. A., Geary, J. C., \& Bowsher, E. C. 2008, Proc. SPIE, 7014, 70142P

Calzetti, D., Armus, L., Bohlin, R. C., Kinney, A. L., Koornneef, J., \& StorchiBergmann, T. 2000, ApJ, 533, 682

Calzetti, D., Kinney, A. L., \& Storchi-Bergmann, T. 1994, ApJ, 429, 582

Eisenhardt, P. R., et al. 2004, ApJS, 154, 48

Elvis, M., et al. 2009, ApJS, 184, 158

Fazio, G. G., et al. 2004, ApJS, 154, 10

Fernández-Soto, A., Lanzetta, K. M., \& Yahil, A. 1999, ApJ, 513, 34

Francis, P. J., Hewett, P. C., Foltz, C. B., Chaffee, F. H., Weymann, R. J., \& Morris, S. L. 1991, ApJ, 373, 465

Frayer, D. T., et al. 2006a, AJ, 131, 250

Frayer, D. T., et al. 2006b, ApJ, 647, L9

Frayer, D. T., et al. 2009, AJ, 138, 1261

Frost, M. J., Surace, J. A., Moustakas, L. A., \& Krick, J. E. 2009, ApJ, 698, L68

Giacconi, R., et al. 2001, ApJ, 551, 624

Giacconi, R., et al. 2002, ApJS, 139, 369

Giavalisco, M., et al. 2004, ApJ, 600, L93

Gordon, K. D., et al. 2007, PASP, 119, 1019

Grazian, A., et al. 2006, A\&A, 449, 951

Hund, L. B., Ashby, M. L., Hora, J. L., \& Surace, J. 2006, BAAS, 38, 1095

Ilbert, O., et al. 2009, ApJ, 690, 1236

Kinney, A. L., Calzetti, D., Bohlin, R. C., McQuade, K., Storchi-Bergmann, T., \& Schmitt, H. R. 1996, ApJ, 467, 38

Krick, J. E., Surace, J. A., Thompson, D., Ashby, M. L. N., Hora, J. L., Gorjian, V., \& Yan, L. 2008, ApJ, 686, 918

Krick, J. E., Surace, J. A., Thompson, D., Ashby, M. L. N., Hora, J. L., Gorjian, V., \& Yan, L. 2009, ApJ, 700, 123

Lacy, M., et al. 2004, ApJS, 154, 166

Laidler, V. G., et al. 2007, PASP, 119, 1325

Laird, E. S., et al. 2009, ApJS, 180, 102

Lejeune, T., \& Schaerer, D. 2001, A\&A, 366, 538

Lonsdale, C. J., et al. 2003, PASP, 115, 897

Makovoz, D., \& Marleau, F. R. 2005, PASP, 117, 1113

Manners, J. C., et al. 2003, MNRAS, 343, 293 
Murakami, H., et al. 2007, PASJ, 59, 369

Negrello, M., et al. 2009, MNRAS, 394, 375

Ohyama, Y., et al. 2007, PASJ, 59, 411

Onaka, T., et al. 2007, PASJ, 59, 401

Papovich, C., Dickinson, M., \& Ferguson, H. C. 2001, ApJ, 559, 620

Polletta, M., et al. 2007, ApJ, 663, 81

Rieke, G. H., et al. 2004, ApJS, 154, 25

Rix, H.-W., et al. 2004, ApJS, 152, 163

Rowan-Robinson, M., et al. 2008, MNRAS, 386, 697

Sajina, A., Lacy, M., \& Scott, D. 2005, ApJ, 621, 256

Salvato, M., et al. 2009, ApJ, 690, 1250
Scoville, N., et al. 2007, ApJS, 172,

Shapley, A. E., Steidel, C. C., Erb, D. K., Reddy, N. A., Adelberger, K. L., Pettini, M., Barmby, P., \& Huang, J. 2005, ApJ, 626, 698

Stansberry, J. A., et al. 2007, PASP, 119, 1038

Stern, D., et al. 2005, ApJ, 631, 163

Surace, J. A., Shupe, D. L., Fang, F., Evans, T., Alexov, A., Frayer, D., Lonsdale, C. J., \& SWIRE Team, 2005, BAAS, 37, 1246

Surace, J. A., et al. 2007, BAAS, 38, 93

Werner, M. W., et al. 2004, ApJS, 154, 1

Williams, R. E., et al. 1996, AJ, 112, 1335

Wilson, J. C., et al. 2003, Proc. SPIE, 4841, 451 